\title{
GEOMETRIC EVALUATION OF ROAD SIGNS USING RADIOMETRIC INFORMATION FROM LASER SCANNING DATA
}

\author{
Higinio González-Jorge*, Belén Riveiro, Julia Armesto, and P. Arias \\ Department of Natural Resources and Environmental Engineering. University of Vigo, Vigo, Spain \\ *Corresponding author (higiniog@uvigo.es)
}

\begin{abstract}
Road signs appear as one of the most important elements for safety purposes and their inspection is typically included in the road management systems. Nowadays, it is becoming more common the use of high performance vehicles to improve the efficiency of the road inspection works. They include sensors as laser scanning for geometric measurements, optical profilers for the detection of cracks on pavement, ground penetrating radar for measuring pavement thickness and video cameras for the visual reference.
\end{abstract}

The road signs use reflective paints to maximize the visibility by the drivers. This coating produces a high reflected radiation in the photoelectric detector of the laser scanners, which allows establishing a simple filter for the 3D segmentation of the road sign.

In this work, a number of road signs are evaluated using a laser scanner Riegl LMS Z390i. The geometrical parameters under study were the sign flatness and the tilt angle with the plane of the pavement. A Matlab algorithm is developed for all the data processing (3D segmentation and evaluation of geometric parameters). The algorithms developed open the possibility to use laser scanner retroreflectivity for segmentation purposes in the evaluation of the condition state of road signs and could increase the productivity of the inspection works.

Keywords: Road Inspection, Laser Scanning, Radiance, Road Sign

\section{INTRODUCTION}

Safety on the roads has become an issue of vital importance, social concern and widespread media coverage in our days. The danger to the road users is reduced by implementing three complementary strategies. The first one is related with the infrastructure design and maintenance, the second one with the vehicle safety and the third one with the regulations of road users.

The road maintenance is a key factor as the roads deteriorate over time $[1,2]$. Deterioration is primarily due to accumulated damage from vehicles and environmental effects (i.e. frost heaves, thermal cracking, oxidation). Maintenance works are supported in three basic stages: inspection of the road, evaluation of the condition state and decision-making and development of rehabilitation if necessary. The rehabilitation works include treatments for asphalt concrete (i.e. crack sealing, micro-milling and surface treatments), slab stabilization, joint sealing, redraw of line markings, replacement of damaged traffic signs, etc.

During the last years high-performance vehicles are more and more used for the automated inspection of the roads. Such vehicles include sensors like surface profilers to the measurement of the International Roughness Index (IRI), the Ride Number (RN), rut depth and macro texture, friction testers that provide the continuous coefficient of friction, road surface deflectometers to evaluate the structural response of the pavement, ground penetrating radar that allows estimating the pavement thickness, laser scanners to acquire data of transverse profiles, bridge 
heights and vertical and horizontal clearances, digital terrain models, tunnel survey, ground movement monitoring, etc. [3]

Laser scanners are ideally suited for mobile surveying and mapping applications. They provide a large amount of data in a very short period of time. These systems typically operate in conjunction with digital cameras that allow obtaining colored point clouds. However, this technology makes necessary to develop technical procedures and algorithms for data processing in order to automatically obtain the required information from the road.

Laser scanner systems typically provide information about geometry and radiometry, in addition to that generated by the associated cameras. The radiation that reaches the phototodetector of the scanner depends of several aspects such as distance to the object, wavelength, environmental conditions, incidence angle on the object surface, and the own reflectivity of the surface [4].

This work shows a technical procedure for the evaluation of the condition state of the road signs using the geometric and radiometric information obtained from a laser scanner system.

\section{EXPERIMENTAL}

\subsection{AREA OF STUDY}

The Rías Baixas A-52 is a highway between the towns of Benavente (Zamora) and O Porriño (Pontevedra), both located in Spain. It is $306 \mathrm{~km}$ long and connects the provinces of Pontevedra, Ourense and Zamora with Madrid. Its construction works began in 1994 and ended on 1998. The work proved more costly than the initially expected due to the complex orography of the provinces of Ourense and Pontevedra and landslides and terrain difficulties continuously appear. The connection between the city center of Ourense $(\mathrm{N}-120)$ and the highway is done through an entrance that is particularly dangerous because of the mountainous terrain and the presence of a river, so the speed is limited to avoid car accidents (Figure 1) by an important number of traffic signs. The traffic signs suffer degradation (dirt substances, wind storms, vandalism, water damage, concealment by vegetation, car accidents, etc) which may provoke a decreasing in the visibility of drivers and contribute to increase the danger of the road.
Figure 2 shows an example of different traffics signs and their associated damages.

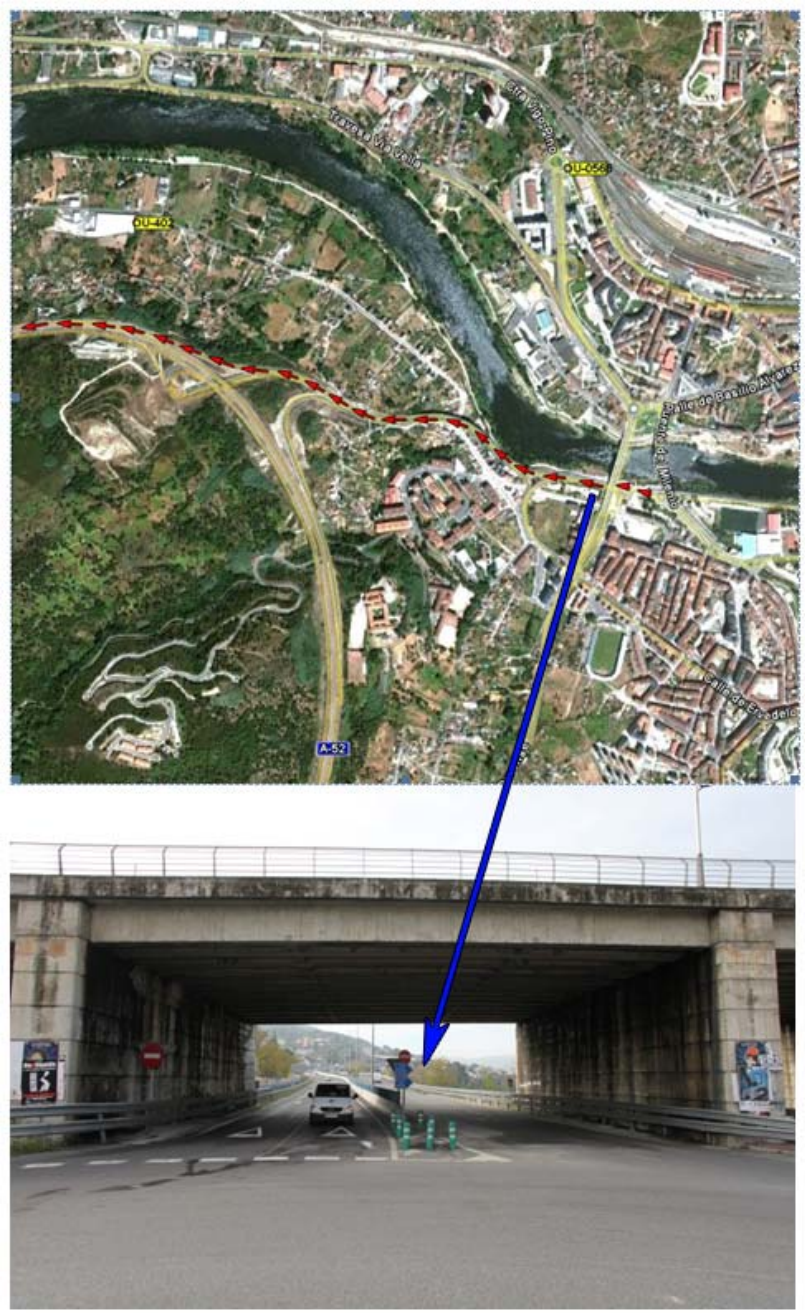

Fig. 1. Connection between the Rias Baixas A-52 and the city center of Ourense.

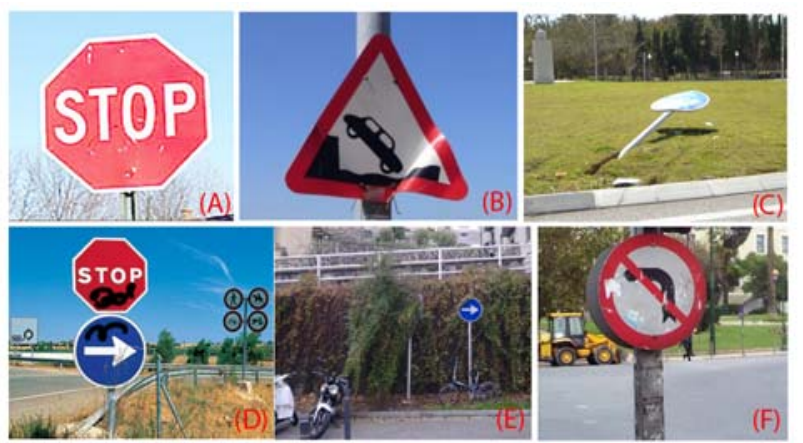

Fig. 2. Different examples of damages in traffic signs: (A) paint deterioration, (B) folded sheet, (C) pole bent, (D) vandalism, $(\mathrm{E})$ concealment by vegetation and $(\mathrm{F})$ adhered substances. 


\subsection{TERRESTRIAL LASER SCANNER}

The terrestrial laser scanning system RIEGL LMS-Z390i is composed of an accurate and fast 3D scanner with the associated software RISCAN PRO. The RIEGL LMSZ390i (Figure 3) is a rugged and fully portable sensor especially designed for the rapid and accurate acquisition of high-quality three dimensional images and particularly indicated for architecture and façade measurement, archaeology and cultural heritage documentation, civil engineering, city modeling and surveying.

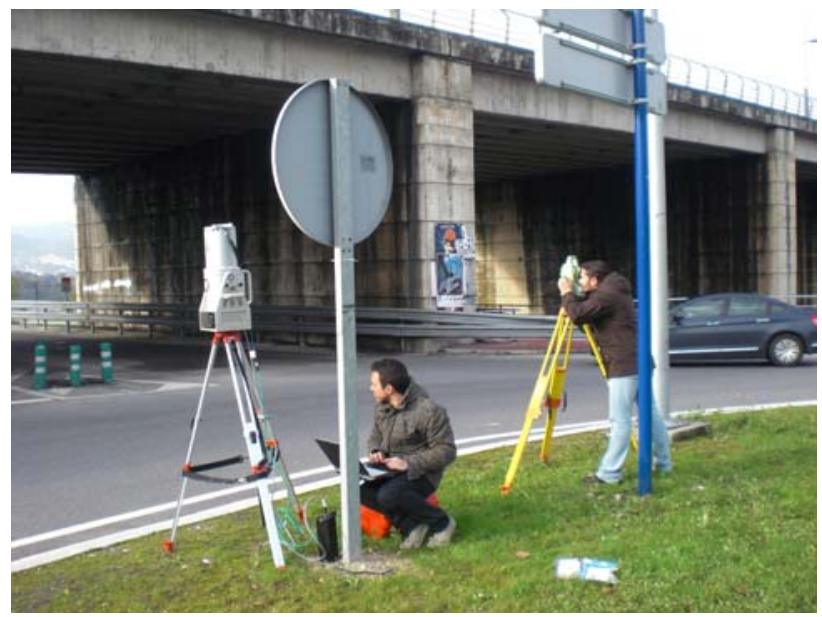

Fig. 3. RIEGL LMS Z390i during surveying process.

Measurements are performed using the time of flight principle for range and two encoders for angular evaluation. Time of flight scanners use a diode pumped laser and the distance is based on the return flight time of each laser beam. There are other technologies in the market based on the phase difference of the coming beam. Time of flight scanners are more indicated for long ranges, but they have a higher signal to noise ratio in short and medium range. Precision mechatronics systems are used for positioning the mirrors which orientate the laser beam to the measuring field. A rotating mirror with $90^{\circ}$ travel is used for vertical measurement and a servo motor that rotates $360^{\circ}$ allows the horizontal scanning. The point cloud obtained from the Spherical coordinates $(R, \theta, \phi)$ is then directly converted into Cartesian coordinates $(X, Y, Z)$ by the software. Furthermore, the laser scanner used in this experiment includes an infrared class 1 laser which means that is inherently safe and there is no possibility of eye damage. Technical characteristics are shown in Table 1.

\begin{tabular}{|l|l|}
\hline Measurement range & $<400 \mathrm{~m}$ \\
\hline Minimum range & $>1 \mathrm{~m}$ \\
\hline Accuracy (50 m) & $6 \mathrm{~mm}$ \\
\hline Repeatability (50 m) & $4 \mathrm{~mm}$ \\
\hline Measurement rate & $11.000 \mathrm{~Hz}$ \\
\hline Laser wavelength & $1.540 \mathrm{~nm}$ \\
\hline Beam divergence & $0.3 \mathrm{mrd}$ \\
\hline Vertical scanner range & $80^{\circ}$ \\
\hline Horizontal scanner range & $360^{\circ}$ \\
\hline Vertical and horizontal step-width & $0.002^{\circ}$ \\
\hline
\end{tabular}

Table 1 Technical specifications of laser scanner RIEGL LMS Z390i.

\section{RESULTS AND DISCUSSION}

The first step in the data acquisition with the scanner, once it is positioned at the roundabout corresponding to the highway entrance (Figure 3), is to perform an overview scan $-360^{\circ}$ horizontal range and $0.2^{\circ}$ step-width (Figure 4) for situating all the objects around. The scan has 713.216 points and takes 1'29’"

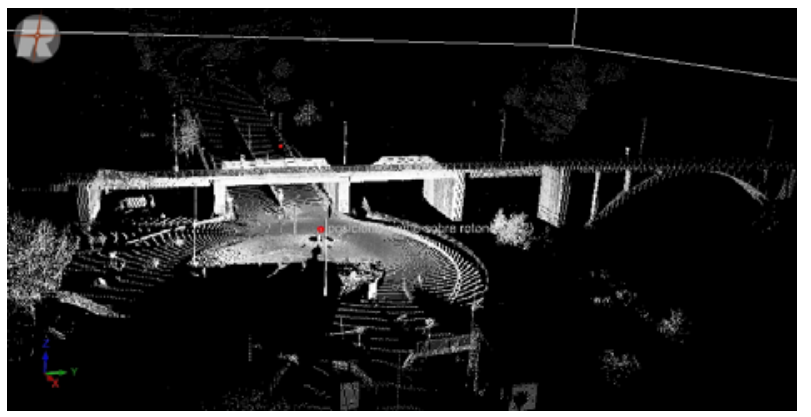

Fig. 4. Overview scan.

The second step is to perform a detailed scan (Figure 5) of the highway entrance. Scan characteristics are a step-width of $0.04^{\circ}, 1.986 .892$ measured points and 8'23’’ of time. 


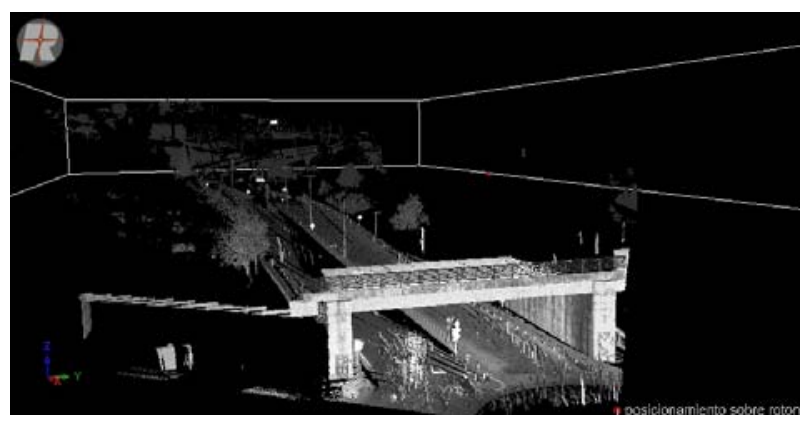

Figure 5. Detailed scan.

The scan is performed in the presence of vehicles circulating in the infrastructure. This causes the existence of echoes in the point cloud attributed to the impact of the laser with such vehicles. These points are identified as dotted lines that can be easily filtered using a disconnected points filter which evaluates the proximity between points and delete the isolated points and dotted lines. Figure 6 shows how the filter works. The echoes from the vehicles are more important as cars get close to the roundabout where the speed of the vehicles is slower.
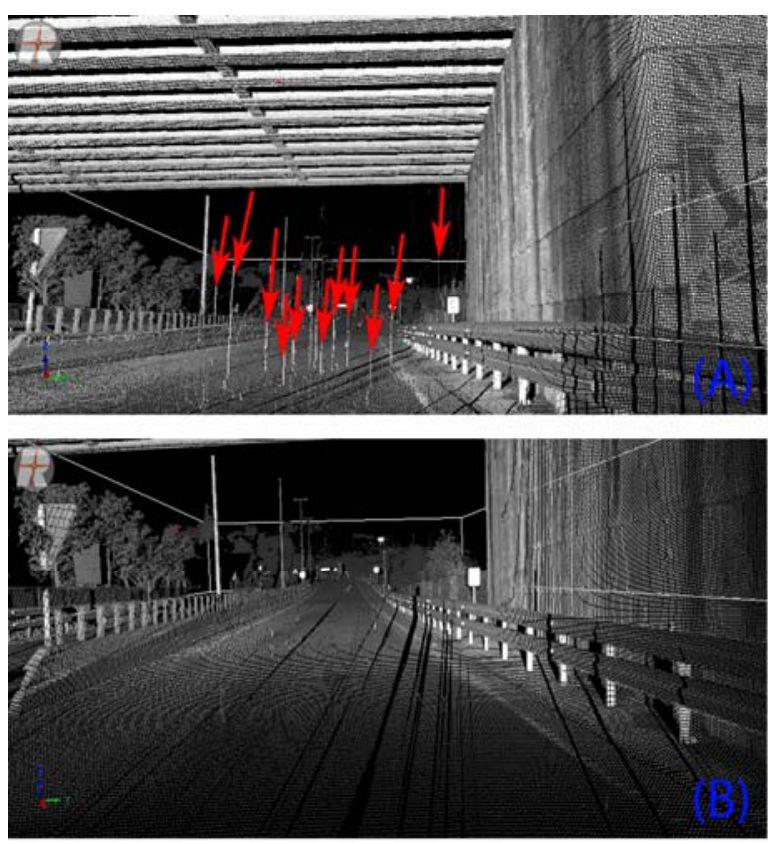

Fig. 6. (A) Echoes from vehicles and (B) point cloud filtered using the disconnected points filter.

The RIEGL scanner assigns radiance values ranging from 0.0 to 1.0 to each of the coordinates of the point cloud.
Radiance with a value of 1.0 means that all the radiation emitted by the laser diode is equal to that which reaches the photodetector. The next step in data processing is to evaluate the retroreflectivity of the different elements located in the road surrounds, mainly the traffic signs under study, and then calibrate the appropriate threshold for the segmentation of the point cloud based on a radiance based filter. This process can be seen in Figure 7. The results show that values between 0.4 and 1.0 (Figure $7 \mathrm{E}$; see highlight) extract all the information related with the signs and avoid the other elements of the road (pavement, vegetation, guardrails, light poles, etc.)

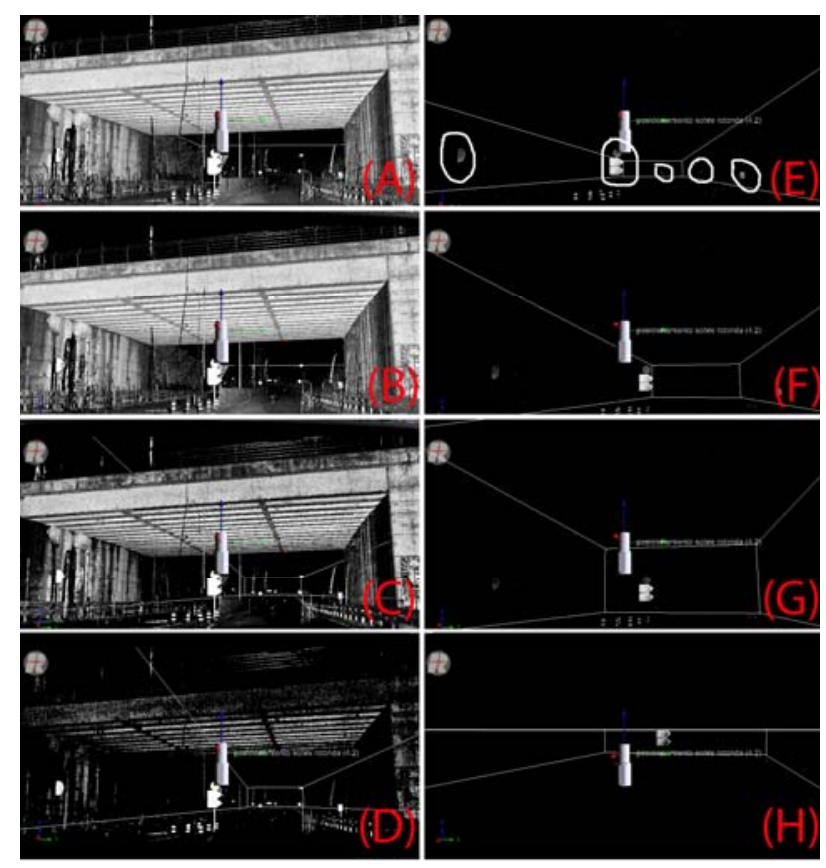

Fig. 7. Calibration of the radiance threshold. (A) $0.0-1.0$, (B) $0.1-1.0$, (C) $0.2-1.0$, (D) $0.3-1.0$, (E) $0.4-1.0$, (F) $0.5-1.0,(\mathrm{G}) 0.6-1.0$ and $(\mathrm{H}) 0.7-1.0$

The last step before to the geometric calculations involves the segmentation of the pavement. This will allow evaluating the angle between the traffic signs and the pavement and automatically determining if a sign is tilted over the predefined tolerance and could make difficult the visualization by the drivers. For this purpose a simple height filter is used. The filter removes all the geometric 
coordinates that are $10 \mathrm{~cm}$ over the pavement plane (Figure

$8)$.

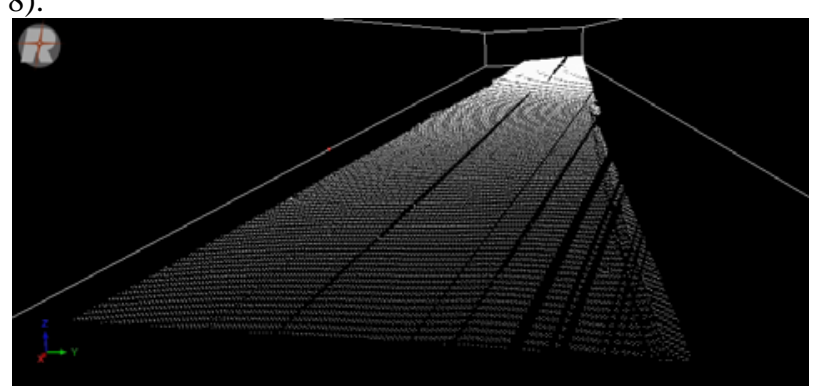

Fig. 8. Pavement segmentation.

Finally, the point cloud from the traffic signs (Fig. 7 E) and the point cloud from the pavement (Fig. 8) are merged on a single point cloud (Figure 9)

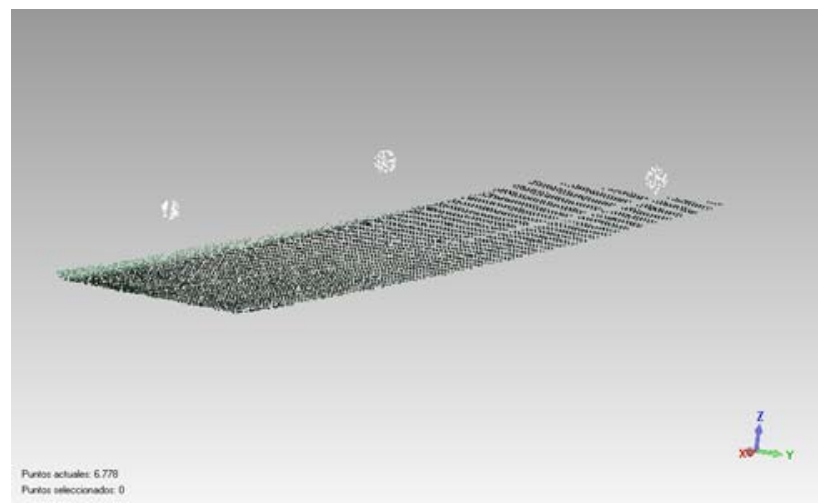

Fig. 9. Merged point cloud including traffic signs and pavement.

Figure 10 shows the different planes fitted to the point clouds merging the data from the traffic signs and from the pavement. The equation of the planes is automatically obtained using a least squares fitting algorithm programmed using Matlab. One pavement section of $10 \mathrm{~m}$ length is used for the evaluation of the pavement plane corresponding to each traffic sign. This strategy avoids the lack accuracy given by the changes in the curvature and slope of the road.

The angle $\alpha$ between the plane fitted to the traffic signs and the plane of the pavement (Table 2) is evaluated using the following equation.

$$
\propto=\frac{180}{\pi} \operatorname{acos}\left(\frac{u_{S} u_{P}+v_{S} v_{P}+w_{S} w_{P}}{\sqrt{u_{S}^{2}+v_{S}^{2}+w_{S}^{2}} \sqrt{u_{P}^{2}+v_{P}^{2}+w_{P}^{2}}}\right)
$$

where $\left(u_{S}, v_{S}, w_{S}\right)$ is the normal vector to the traffic sign, $\left(u_{P}, v_{P}, w_{P}\right)$ the normal vector to the pavement plane.

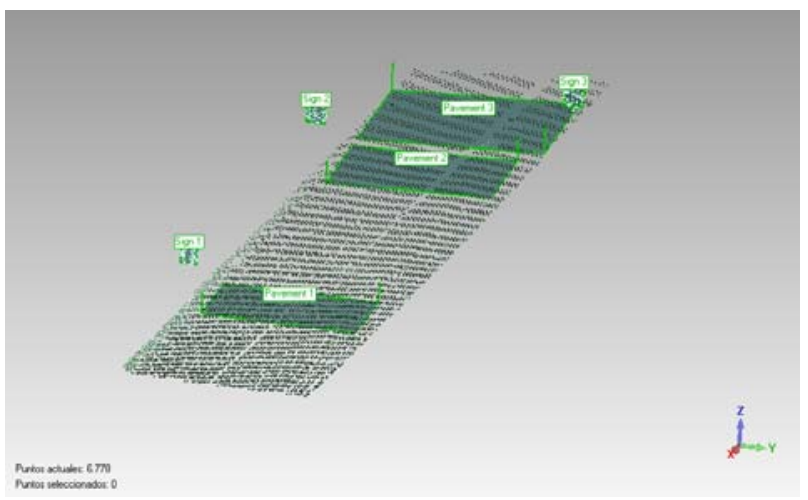

Fig. 10. Planes fitted to pavement and traffic signs.

The plane fitted to the traffic signs also gives important information about the condition state of the sign. Standard deviation values give evidence about the flatness of the sign sheet (Table 2). Figure 11 shows a sign (sign 7) with a folded sheet (Fig. 2 B) which results in a high standard deviation. A similar behavior is also depicted by sign 9 .

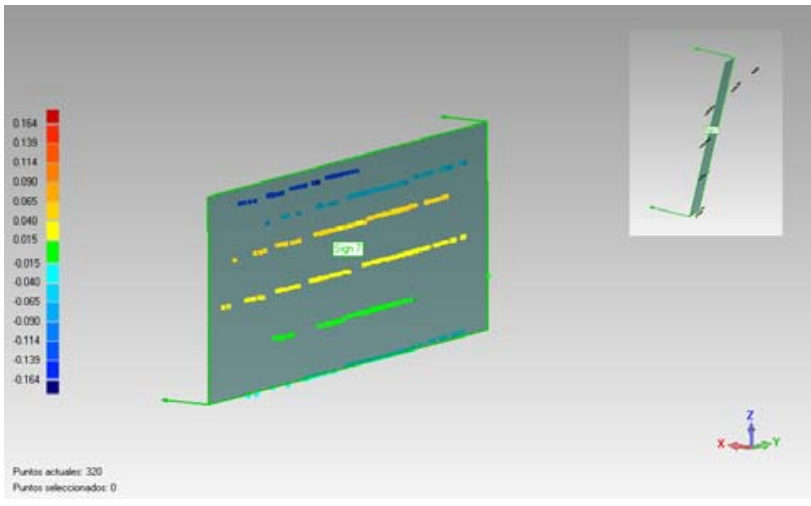

Fig. 11. Deviations of the point cloud from the plane fitted.

Figure 12 show the radiance pattern of the points segmented from one of the traffic signs under study (sign 3). The radiance data are fitted with a least squares regression line (results are shown in the graph, including 
the residuals). Then the standard deviation also appears as an important parameter to evaluate if significant changes in the radiometry appear. For example a traffic sign with some substance adhered to the reflective coating will provoke a decrease in the radiance value of the points that return from that region and will increase the standard deviation of the fitting surface. Table 2 shows the results for all the signs. All of them show a quite similar standard deviation which mean that do not exist substances adhered to the signs or coating degradation. This information was visually corroborated by the recorded photographs.
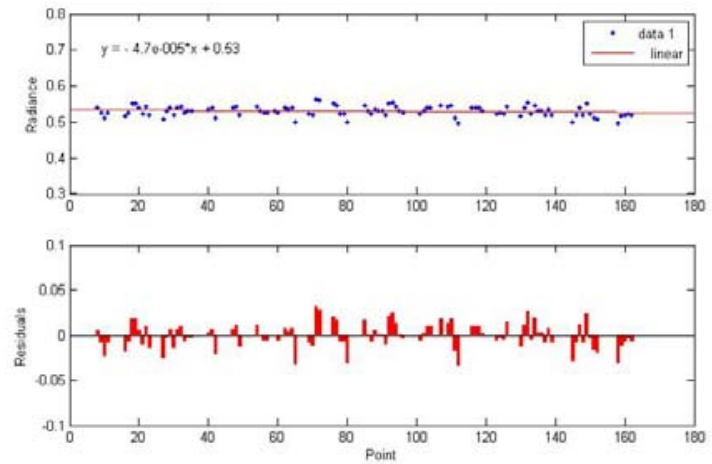

Fig. 12. Radiance results of traffic sign 3.

\begin{tabular}{|c|c|c|c|}
\hline $\begin{array}{c}\text { Sign } \\
\text { number }\end{array}$ & $\boldsymbol{\alpha} \mathbf{(}^{\mathbf{}} \mathbf{)}$ & $\begin{array}{c}\text { Std. - plane } \\
\text { fitting (m) }\end{array}$ & Std. - line fitting \\
\hline 1 & 90.13 & 0.003 & 0.029 \\
\hline 2 & 88.15 & 0.005 & 0.032 \\
\hline 3 & 91.28 & 0.004 & 0.037 \\
\hline 4 & 90.67 & 0.006 & 0.028 \\
\hline 5 & 92.54 & 0.007 & 0.034 \\
\hline 6 & 87.78 & 0.004 & 0.032 \\
\hline 7 & 86.36 & 0.064 & 0.030 \\
\hline 8 & 92.34 & 0.003 & 0.031 \\
\hline 9 & 93.67 & 0.074 & 0.034 \\
\hline
\end{tabular}

Table 2. Summary of the results.

\section{CONCLUSIONS}

The procedure developed for this work uses the data obtained from a laser scanner (terrestrial or mobile) to automatically evaluate some important parameters for determining the condition state of the traffic signs.
The procedure is essentially based on the segmentation of the geometry of the signs from their retroreflectivity. The sign status is evaluated using three different parameters which are also automatically calculated. The first one is based on the angle between the sign and the pavement plane, the second one in the calculation of the sign flatness and the last one in the evaluation of the standard deviation of the sign retroreflectivity.

This work is a contribution to the systematization and to the establishment of rules for the tasks involved in inspection programs and also to improve the safety of the roads.

\section{ACKNOLEDGEMENTS}

Authors would like to give thanks to Consellería de Economía e Industria (Xunta de Galicia) and Ministerio de Ciencia e Innovación (Gobierno de España) for the financial support given; human resources programs (IPP055 - EXP44, FPU AP2006-04663) and projects (INCITE09 TMT051E PR and BIA2009-08012).

\section{REFERENCES}

[1] Vera, I., Thenoux, G., Solminihax, H. D., and Echaveguren, T., “Technical assessment model for the performance of flexible pavement maintenance”, Revista de la Construcción, Vol. 9 (2), pp. 76 - 88, 2010.

[2] Pellegrino, C., Pipinato, A., and Modena C., "A simplified management procedure for bridge network maintenance”, Structure and Infraestructure Engineering, Vol 7 (5), pp. 341 - 351, 2011

[3] Findley, D. J., Cunningham, C. M., and Hummer, J. E., “Comparison of mobile and manual collection for roadway components”, Transportation Research, Vol. 19 (3), pp. 521 - 540, 2011.

[4] Armesto-González, J., Riveiro-Rodriguez, B., González-Aguilera, D., and Rivas-Brea, M. T., “Terrestrial laser scanning intensity data applied to damage detection for historical buildings", Journal of Archaeological Science, Vol. 37 (12), pp. 3027 - 3047, 2010. 